\title{
Lack of pronounced changes in the expression of fatty acid handling proteins in adipose tissue and plasma of morbidly obese humans
}

\author{
Ewa Anna Grzegorczyk @1], Ewa Harasim-Symbor², Bartlomiej Lukaszuk², Dorota Harasiuk², Barbara Choromanska², \\ Piotr Mysliwiec ${ }^{3}$, Malgorzata Zendzian-Piotrowska ${ }^{1}$ and Adrian Chabowski
}

\begin{abstract}
Background/Objectives: Fatty acid handling proteins are involved in the process of accumulation of lipids in different fat tissue depots. Thus, the aim of the study was to estimate the expression of both fatty acid transport and binding proteins in the subcutaneous (SAT) and visceral adipose tissue (VAT) of patients with morbid obesity without metabolic syndrome, as well as the plasma concentrations of these transporters.

Subjects/Methods: Protein (Western blotting) and mRNA (Real-time PCR) expression of selected fatty acid handling proteins was assessed in the visceral and subcutaneous adipose tissue of 30 patients with morbid obesity. The control group consisted of 10 lean age-matched patients. Plasma levels of fatty acid protein transporters were also evaluated using ELISA method. Moreover, total plasma fatty acid composition and concentration was determined by gas-liquid chromatography (GLC).
\end{abstract}

Results: Significant increase in fatty acid translocase (FAT/CD36) mRNA $(P=0.03)$ and plasmalemmal $(P=0.01)$ expression was observed in VAT of patients with morbid obesity vs. lean subjects together with elevation of lipoprotein lipase (LPL), as well as peroxisome proliferator-activated receptor $\gamma$ (PPAR $\gamma$ ) in both examined compartments of adipose tissue. Moreover, in obese subjects plasma concentration of RBP4 was markedly elevated ( $P$ $=0.04)$ and sCD36 level presented a tendency for an increase $(P=0.08)$ with concomitant lack of changes in FABP4 concentration $(P>0.05)$.

Conclusions: Fatty acid transport into adipocytes may be, at least in part, related to the increased expression of FAT/ CD36 in the VAT of morbidly obese patients, which is accompanied by augmented expression of LPL, as well as PPARY. Probably, alternations in plasma concentrations of RBP4 and sCD36 in obese patients are associated with "unhealthy" fat distribution.

Correspondence: Ewa Harasim-Symbor (eharasim@umb.edu.pl)

${ }^{1}$ Department of Hygiene, Epidemiology and Ergonomics, Medical University of Bialystok, Bialystok, Poland

${ }^{2}$ Department of Physiology, Medical University of Bialystok, Mickiewicza 2C, 15222 Bialystok, Poland

Full list of author information is available at the end of the article

\section{Introduction}

Obesity is one of the most serious health problems in many countries and the prevalence of its occurrence is increasing worldwide ${ }^{1}$. Important pathophysiological basis of obesity is an increase in size and number of adipocytes due to the uptake and storage of the excess of energy in the form of lipids ${ }^{2}$. Subcutaneous obesity is

\section{(c) The Author(s) 2017}

(c) Open Access This article is licensed under a Creative Commons Attribution 4.0 International License, which permits use, sharing, adaptation, distribution and reproduction cc) in any medium or format, as long as you give appropriate credit to the original author(s) and the source, provide a link to the Creative Commons license, and indicate if changes were made. The images or other third party material in this article are included in the article's Creative Commons license, unless indicated otherwise in a credit line to the material. If material is not included in the article's Creative Commons license and your intended use is not permitted by statutory regulation or exceeds the permitted use, you will need to obtain permission directly from the copyright holder. To view a copy of this license, visit http://creativecommons.org/licenses/by/4.0/. 
referred to peripheral fat accumulation, whereas visceral obesity is generally related to abdominal fat accumulation within omental and mesenteric fat depots. Epidemiological studies have shown that visceral obesity compared to peripheral obesity is associated with a higher risk of obesity-related comorbidities such as insulin resistance, type 2 diabetes, cardiovascular disease, and dyslipidemia ${ }^{3}$.

Fatty acid (FA) transport into adipocytes apart from simple diffusion is a protein-mediated process. Protein transporters that were found to be highly involved in the facilitation of fatty acid uptake in adipose tissue are ${ }^{4}$ fatty acid translocase (FAT/CD36), plasmalemmal fatty acid binding protein (FABPpm), fatty acid transport protein (FATP-4), and cytosolic adipocyte fatty acid binding protein (FABP4). FAT/CD36 was identified as a key longchain fatty acid (LCFA) transporter in fat tissue ${ }^{5}$. Furthermore, it was shown that FABPpm is expressed in a wide variety of tissues and its expression is up-regulated during preadipocyte differentiation. Other fatty acid transport proteins are also implicated in the FA accumulation in adipocytes. For instance, FATP-4 is known to affect triacylglycerols (TAGs) droplet size and other complex lipid pools formation. Once inside, fatty acids are bound by cytosolic fatty acid binding protein (FABPc), which stimulates not only FA absorption, but also its cytoplasmic redistribution ${ }^{6}$. FABP4 is the most abundant cytosolic isoform in adipocytes, which controls intracellular fatty acid transport and subsequent metabolism in fat tissue. The relative content of FABP4 in humans varies in different fat tissue depots and it has been shown that its mRNA level is related to the circulating insulin concentration in obese subjects ${ }^{7}$.

However, so far there are no data available on the expression of fatty acid handling proteins, which are involved in the accumulation of lipids in the visceral adipose tissue (VAT) and subcutaneous adipose tissue (SAT) of morbidly obese patients without metabolic syndrome. It is known that adipose tissue serves as a buffer for elevated consumption of dietary fatty acids. We suspect that the expression and/or content of LCFA protein transporters would be enhanced in different compartments (i.e., adipocytes and plasma) in order to compensate increased availability of lipids, and subsequently accumulate them as storage fraction (i.e., TAG) in adipocytes. Thus, the aim of our study was to assess the expression of membrane fatty acid handling proteins (FAT/CD36, FABPpm, FATP-4) at the mRNA and protein level in the SAT and VAT of patients with obesity, as well as the expression of lipoprotein lipase (LPL), peroxisome proliferator-activated receptor $\gamma$ (PPAR $\gamma$ ), cytosolic FABP4 and FABP5. Furthermore, the plasma concentrations of soluble CD36 (sCD36), FABP4 and retinol binding protein (RBP4) along with total plasma fatty acid composition have been determined.

\section{Materials and methods}

The study included 30 obese patients $(B M I>40)(24$ women and 6 men) without diabetes, hypertension, or other components of metabolic syndrome. General characteristic of patients are described in the Table 1 . The individuals underwent bariatric surgery due to morbid obesity (BMI > 40). Control group consisted of 10 lean age-matched patients $(\mathrm{BMI} \leq 26)(7$ women and $3 \mathrm{men})$, who underwent elective laparoscopic cholecystectomy. All patients gave their informed consent to participate in the study. Moreover, all experiments were conducted in accordance with the guidelines of the Ethical Committee at the Medical University of Bialystok. Patients with acute inflammatory diseases and history of malignancy were excluded from the study. All the subjects were treated at the Department of General and Endocrinological Surgery of the University Hospital in Bialystok.

Fasting blood samples were taken from control subjects and patients with obesity before surgery and were collected at EDTA-coated tubes. Next, the blood was centrifuged (10 min, 4000 r.p.m.) and the resultant samples were stored at $-80^{\circ} \mathrm{C}$ until further measurements. All parameters included in Table 1 were determined using routine methods in the certified clinical laboratories of the University Hospital in Bialystok (i.e., Department of Biochemical Diagnostics and Department of Hematological Diagnostics). At the end of the surgical intervention, the samples of SAT and VAT were taken from the upper part of the abdomen and promptly frozen in liquid nitrogen and stored at $-80^{\circ} \mathrm{C}$.

\section{Real-time PCR}

Total RNA was isolated from $30 \mathrm{mg}$ of frozen tissue using NucleoSpin RNA (Macherey-Nagel) according to the producer's instructions. First strand cDNA was generated using High Capacity cDNA Reverse Transkription Kit (Applied Biosystem). PCR products were obtained by amplification of cDNA using primers, as follows: forward: 5'-GGACGCTGAGGACAACAC-3'; reverse: 5'-GCCAGATTGAGAACTGTGAAG-3' for FAT/CD36; forward: 5'-TAAGTTCAGCCGAGATGTC-3'; reverse: 5'-GTCATAATACCGATAACCTTGTAG-3' for FABPpm; forward: 5'- GAAGGCAAAGGTGCGACAGT-3'; reverse: 5'- GCCGAACGGTAGAGGCAAA-3' for FATP-4; forward: 5'-ATGTTCGTCATGGGTGTGAA-3'; reverse: $5^{\prime}$ GGTGCTAAGCAGTTGGTGGT-3' for GAPDH. PCR was performed with SYBR Green JumpStart Taq ReadyMix (Sigma), using Bio-Rad Chromo4 system. Reaction mix in final volume of $25 \mu \mathrm{l}$ consisting of $12,5 \mu \mathrm{l}$ SYBR Green I, $3 \mu \mathrm{l} \mathrm{cDNA}$, and $300 \mathrm{nM}$ of each primer pairs. PCR was carried out under the following conditions: $15 \mathrm{~s}$ denaturation at $54{ }^{\circ} \mathrm{C}, 1 \mathrm{~min}$ annealing at $56^{\circ} \mathrm{C}$ for FABPpm and GAPDH, $58^{\circ} \mathrm{C}$ for FAT/CD36, $62^{\circ} \mathrm{C}$ for FATP-4, $1 \mathrm{~min}$ extension at $72{ }^{\circ} \mathrm{C}$ for 40 cycles. PCR 
Table 1 General characteristic of obese and lean patients

\begin{tabular}{|c|c|c|c|}
\hline Parameter & Lean patients & Obese patients & Reference values \\
\hline Age & $42,1 \pm 10,5$ & $46,3 \pm 11,9$ & - \\
\hline WHR & $0,82 \pm 0,1$ & $0,93 \pm 0,09$ & Male $<0,9 ;$ Female $<0,85$ \\
\hline BMI (kg/m2) & $24,5 \pm 3,1$ & $42,8 \pm 5,9$ & $18,5-24,99$ \\
\hline CRP (mg/l) & $5,7 \pm 3,2$ & $9,5 \pm 5,3$ & $(<10)$ \\
\hline Glucose (mg/l) & $98,2 \pm 18,0$ & $100,4 \pm 18,6$ & $(70-115)$ \\
\hline $\operatorname{ALT}(U \mathrm{I} / \mathrm{l})$ & $26,9 \pm 9,8$ & $27,2 \pm 10,1$ & $(5-40)$ \\
\hline AST (UI/I) & $22,1 \pm 6,0$ & $21,0 \pm 6,3$ & $(5-40)$ \\
\hline Cholesterol (mg/l) & $190,2 \pm 29,1$ & $199,0 \pm 35,1$ & $(<200)$ \\
\hline LDL (mg/l) & $104,0 \pm 20,2$ & $128,6 \pm 29,8$ & $(<135)$ \\
\hline TAG (mg/l) & $120,2 \pm 50,3$ & $131,1 \pm 60,4$ & $(50-200)$ \\
\hline $\mathrm{HDL}(\mathrm{mg} / \mathrm{l})$ & $50,1 \pm 8,7$ & $51,1 \pm 9,9$ & $(35-70)$ \\
\hline WBC $(103 / \mu l)$ & $6,2 \pm 1,1$ & $8,2 \pm 1,6$ & $(4,4-11,3)$ \\
\hline RBC $(106 / \mu l)$ & $4,6 \pm 0,5$ & $4,7 \pm 0,4$ & $(4,0-5,6)$ \\
\hline $\mathrm{HGB}(\mathrm{g} / \mathrm{dl})$ & $14,0 \pm 1,1$ & $13,7 \pm 1,2$ & $(12-16)$ \\
\hline HCT (\%) & $39,1 \pm 3,2$ & $41,0 \pm 3,5$ & $(34-47)$ \\
\hline 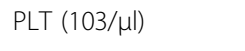 & $289,7 \pm 40,3$ & $303,8 \pm 51,8$ & $(140-440)$ \\
\hline Fibrynogen (mg/dl) & $315,2 \pm 41,4$ & $410,8 \pm 58,0$ & $(170-400)$ \\
\hline INR & $1,0 \pm 0,06$ & $0,9 \pm 0,06$ & $(0,9-1,3)$ \\
\hline
\end{tabular}

All parameters were determined using standard methods in the certified clinical laboratories of the University Hospital in Bialystok

efficiency was examined by serial dilution of the template cDNA, and a melting curve was assessed after each reaction to verify $\mathrm{PCR}$ product specificity. A sample containing no cDNA was used as a negative control to verify the absence of primer dimmers. The results were normalized to GAPDH expression measured in each sample. Relative expression of genes was calculated according to Pfaffl method ${ }^{8}$.

\section{Isolation of plasma membrane fraction}

Plasma membranes were isolated from adipose tissue according to Gargiulo et al. ${ }^{9}$. Briefly, samples of adipose tissue $(250 \mathrm{mg}$ ) was placed in $2.5 \mathrm{ml}$ of ice-cold SHB buffer (20 mM Tris, $1 \mathrm{M}$ sucrose, $1 \mathrm{mM}$ EDTA) containing protease and phosphatase inhibitor cocktail, as well as Triton-X100 (Sigma). The samples were homogenized in a Teflon-Glass homogenizer at $4{ }^{\circ} \mathrm{C}$ and then centrifuged $\left(10 \mathrm{~min}, 500 \mathrm{~g}, 4^{\circ} \mathrm{C}\right)$. Thereafter, the supernatant was layered on sucrose cushion ( $20 \mathrm{mM}$ Tris, $1.12 \mathrm{M}$ sucrose, $1 \mathrm{mM}$ EDTA) and centrifuged ( $25 \mathrm{~min}, 101,000 \mathrm{~g}, 4^{\circ} \mathrm{C}$ ). Next, the pellet containing the plasma membrane fraction was suspended in SHB buffer and collected for Western blot analysis.

\section{Western blot analysis}

Routine Western blotting procedures were used to detect protein content as described ${ }^{10}$. Briefly, the samples were homogenized in ice-cold RIPA (radioimmunoprecipitation assay) buffer containing phosphatase and protease inhibitors (Roche Diagnostics $\mathrm{GmbH}$, Mannheim, Germany). Protein concentration was determined using bicinchonic acid method (BCA) with bovine serum albumin (BSA) as a standard. Next, homogenates were reconstituted in Laemmli buffer, separated by $10 \%$ sodium dodecyl sulfate-polyacrylamide gel electrophoresis, transferred onto nitrocellulose membranes and blocked ( $5 \%$ of nonfat dry milk or BSA). Then, the membranes were incubated overnight at $4{ }^{\circ} \mathrm{C}$ with the corresponding antibodies i.e., FAT/CD36 (1:1000, Abcam, UK), FATP-4 (1:500, Santa Cruz Biotechnology, USA), FABP4 (1:500, Santa Cruz Biotechnology, USA), FABP5 (1:500, Abcam, UK), FABPpm (1:1000, Abcam, UK), LPL (1:1000, Abcam, UK), PPAR $\gamma$ (1:500, Santa Cruz Biotechnology, USA), $\mathrm{Na}^{+} / \mathrm{K}^{+}$pump (1:500, Santa Cruz Biotechnology, USA) and $\beta$-actin (1:500, Cell Signaling Technology, USA). Thereafter, the membranes were incubated with appropriate secondary antibodies conjugated with horseradish peroxidase (Santa Cruz Biotechnology, USA). Protein bands were visualized using an enhanced chemiluminescence substrate (Thermo Scientific, Rockford, IL, USA) and quantified densitometrically (ChemiDoc visualization system EQ, Biorad, Warsaw, Poland). Equal protein concentrations $(50 \mu \mathrm{g})$ were loaded 
in each lane, which was confirmed by Ponceau S staining. Protein expression was normalized to $\beta$-actin. Finally, the control was set to $100 \%$ and the experimental groups were expressed relatively to the control.

\section{Plasma lipid composition}

Measurement of total plasma fatty acid composition was performed according to method described in details by Glaser et al. ${ }^{11}$. Briefly, lipids in the presence of internal standard were extracted from plasma $(250 \mu \mathrm{l})$ based on Folch method ${ }^{12}$ in chloroform/methanol $(2: 1, v / v)$ solution. Thereafter, methanolic $\mathrm{HCl}$ was added and samples were heated up to $85^{\circ} \mathrm{C}$ for $45 \mathrm{~min}$ in order to synthetize methyl esters. After cooling, $1 \mathrm{ml}$ of hexane was added, samples were centrifuged ( $900 \mathrm{~g}, 5 \mathrm{~min}$ ), and upper phase was transferred to a new glass tube. Next, the extract was dried under nitrogen flow and lipids were dissolved in 50 $\mu \mathrm{l}$ of hexane. Individual fatty acid methyl esters were identified and quantified according to the retention times of standards by GLC (Hewlett-Packard 5890 Series II gas chromatograph, HP-INNOWax capillary column).

\section{ELISA analysis}

Concentrations of sCD36, FABP4, and RBP4 were measured using commercially available kits for enzymelinked immunoassay. All procedures were performed following the manufacturer's instruction. sCD36 plasma concentration $(\mathrm{ng} / \mathrm{ml})$ was determined using ELISA kit provided by Aviscera Bioscience Inc. (USA). Eight fold dilution of each sample was required before the assay. Intra-assay and inter-assay coefficients of variations were less than $4-6 \%$ and less than $8-12 \%$, respectively. Plasma concentration of the FABP4 $(\mathrm{ng} / \mathrm{ml})$ was measured using kit supplied by USCN Life Science Inc. (Wuhan, China). Intra-assay and inter-assay coefficients of variations were less than $10 \%$ and less than $12 \%$, respectively. The RBP4 $(\mu \mathrm{g} / \mathrm{ml})$ ELISA kit was also provided by USCN Life Science Inc. (Wuhan, China). Intra-assay and inter-assay coefficients of variations were less than $10 \%$ and less than $12 \%$, respectively. At the end of each measurement the intensity of colored product was measured in a hybrid multi-mode microplate reader (Synergy $\mathrm{H}^{\mathrm{TM}}$, BioTek Instruments, USA) at $450 \mathrm{~nm}$.

\section{Statistical analysis}

The results were statistically analyzed using Statistica 10 (StatSoft, Krakow, Poland). Data were analyzed using oneway analysis of variance (ANOVA) followed by post hoc Tukey's test for groups with the normal distribution and variance homogeneity. Otherwise non-parametric Kruskal-Wallis with subsequent Wilcoxon's pairwise test with Holm correction was applied. Statistical difference was indicated by a $P$-values $<0.05$. Data were presented as mean or fold change \pm SEM.

\section{Results}

Protein and mRNA expression of fatty acid transport proteins (FAT/CD36, FABPpm, and FATP-4)

In VAT, FAT/CD36 mRNA expression was significantly increased in patients with morbid obesity $(+47 \%, P=$ 0.033; Fig. 1a) compared to the lean control. In SAT the expression of FAT/CD36 mRNA showed just a trend towards an increase in patients with obesity $(+31 \%, P=$ 0.067; Fig. 1a). Moreover, we observed a tendency for an increase in the total protein expression of FAT/CD36 in VAT of patients with obesity $(+23 \%, P=0.075$; Fig. $1 \mathrm{~b})$ compared to the lean patients' VAT tissue. Additionally, FAT/CD36 plasmalemmal expression was also significantly elevated in obese VAT tissue $(+51 \%, P=0.019$; Fig. 1c) in comparison with lean subjects.

In contrast to FAT/CD36, we noticed a considerable decrease in the expression of FABPpm mRNA in obese SAT $(-28 \%, P=0.049$; Fig. $2 \mathrm{a})$, as well as obese VAT $(-32 \%, P=0.039$; Fig. 2a) compared with the respective tissues in the control group. Nevertheless, there was no significant difference in the total expression of FABPpm at a protein level in both, the obese SAT and VAT $(P=0.1$ and $P=0.13$; Fig.2c) compared to the respective tissues in the control group.

In contrast to the above mentioned proteins, the expression of FATP-4 mRNA remained unchanged in both SAT and VAT of patients with obesity $(P=0.65$ and $P=0.37$; Fig. $2 b)$ in comparison with the respective tissues in the control group. Thus, protein expression of FATP-4 in the tissues from patients with obesity was not altered compared to the lean patients' SAT, as well as VAT $(P=0.14$ and $P=0.1$; Fig. $2 \mathrm{~d})$.

\section{Protein expression of cytosolic fatty acid binding proteins (FABP4 and FABP5), LPL and PPARY}

The expression of FABP4 remained unchanged in both SAT and VAT of the obese individuals compared with the corresponding tissues of the control group $(P>0.05$; Fig. 3a). Nonetheless, the expression of FABP5 increased significantly in VAT of patients with morbid obesity compared to their SAT level $(+36 \%, P<0.05$; Fig. $3 b)$. Moreover, total expression of LPL, as well as PPAR $\gamma$ was markedly elevated in both SAT $(+234 \%, P=0.002$; Fig. 3c and $+88 \%, P=0.001$; Fig. $3 \mathrm{~d}$, respectively) and VAT $(+191 \%, P=0.005$; Fig. $3 \mathrm{c}$ and $+90 \%, P=0.023$; Fig. $3 \mathrm{~d}$, respectively) adipose tissue in obese individuals compared to control group.

\section{Plasma content of fatty acid binding proteins (FABP4,} RBP4, and SCD36), as well as total fatty acid composition

We did not observe any difference in the plasma concentration of cytosolic FABP4 in subjects with obesity compared to the lean ones $(P=0.09$; Fig. $4 \mathrm{a})$. Moreover, there was a trend towards an increase $(+22 \%, P=0.07$; 


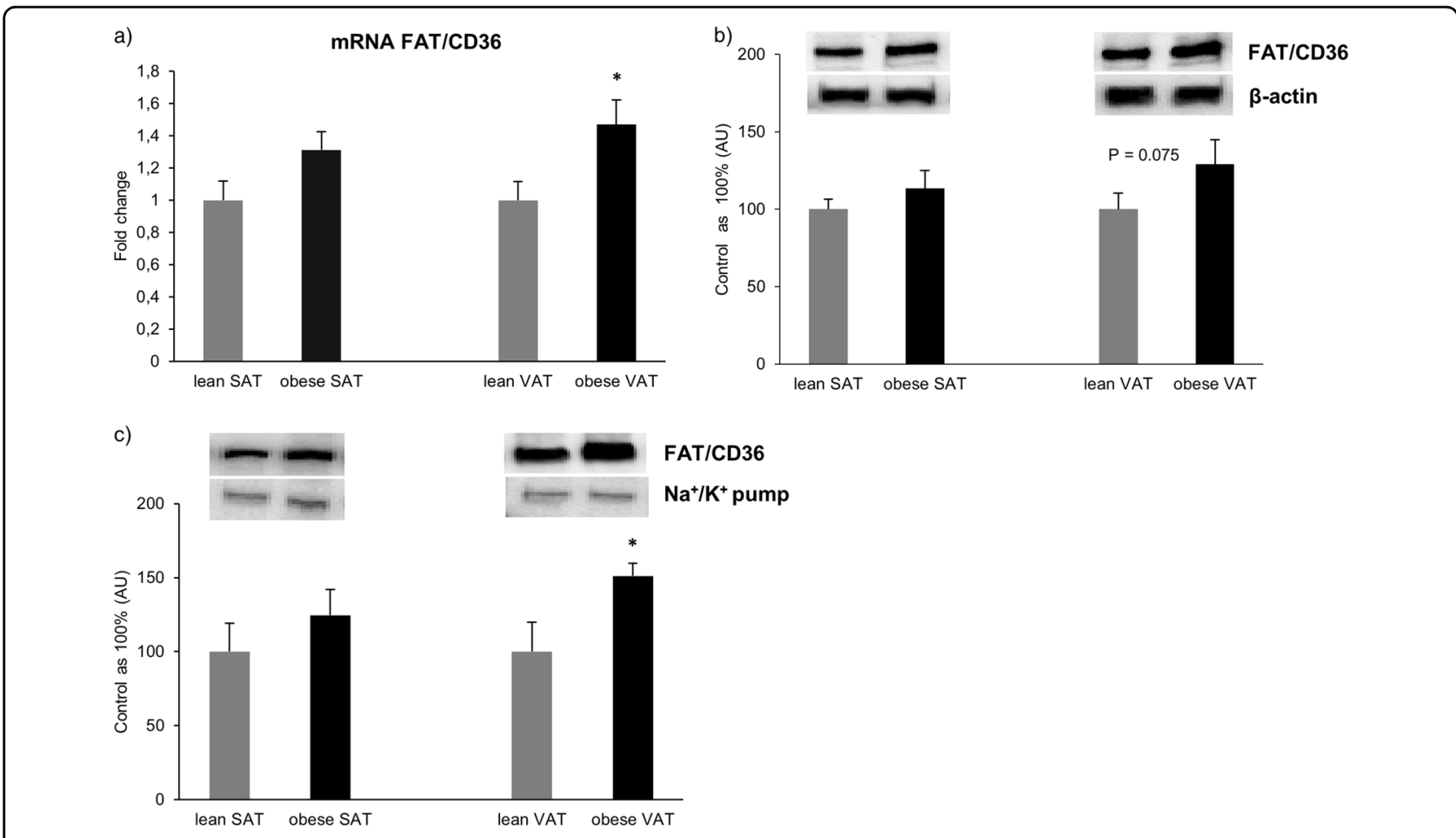

Fig. 1 Expression of FAT/CD36 at the level of mRNA. a as well as total protein (b) and plasma membranes (c) in subcutaneous and visceral adipose tissue of obese and control individuals. The data are expressed as fold change or percentage \pm SEM. Control group was set as $100 \%$ (b) and (c). ${ }^{*} P<0.05$ significant difference vs. respective control group (lean SAT or VAT)

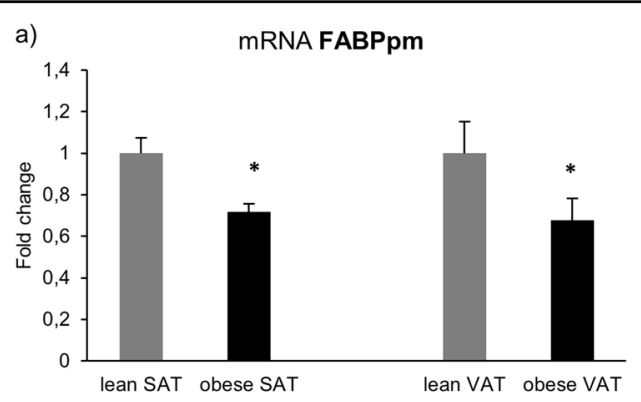

c)

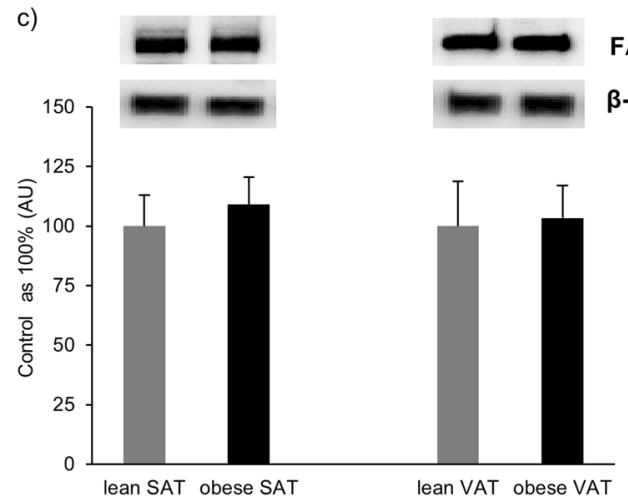

b)
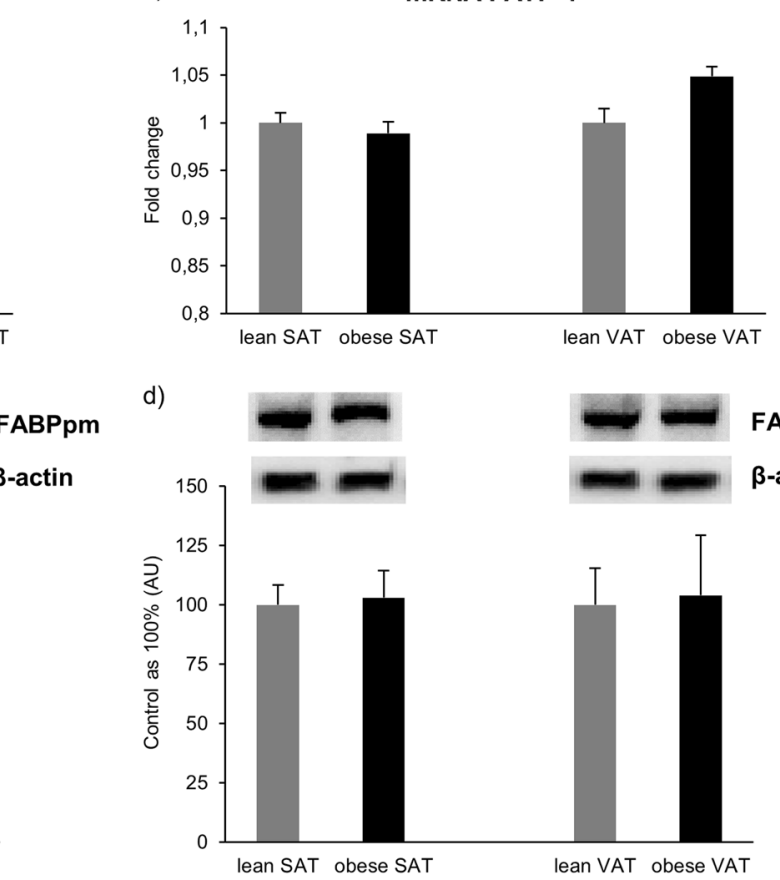

d)
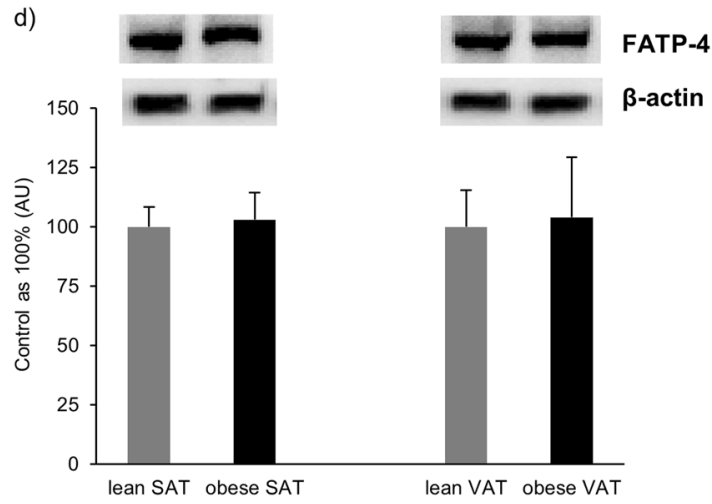

Fig. 2 Expression of FABPpm and FATP-4 at the level of mRNA. $\mathbf{a}, \mathbf{b}$ as well as total protein (c), $\mathbf{d}$ in subcutaneous and visceral adipose tissue of obese and control individuals. The data are expressed as fold change or percentage \pm SEM. Control group was set as $100 \%$ (c) and (d). ${ }^{*} P<$ 0.05 significant difference vs. respective control group (lean SAT or VAT) 

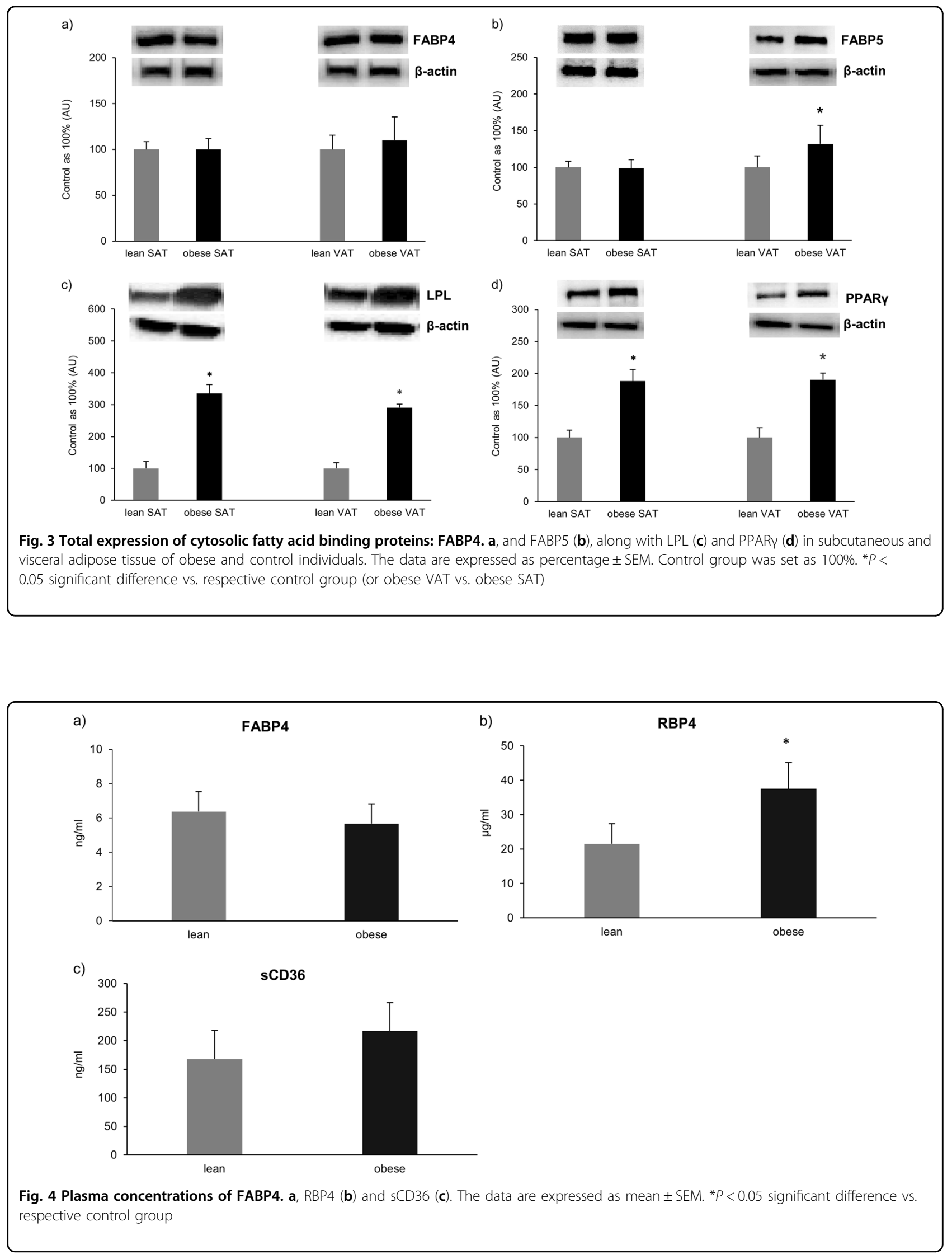
Table 2 Plasma total (from all lipid fractions) and individual fatty acids concentration (milligrams per liter) in obese and lean patients

\begin{tabular}{lll}
\hline Fatty acid & Lean patients & Obese patients \\
\hline Myristic acid (C14:0) & $25,02 \pm 9,21$ & $22,69 \pm 9,32$ \\
Palmitic acid (C16:0) & $687,58 \pm 104,52$ & $738,65 \pm 162,37$ \\
Palmitoleic acid (C16:1) & $68,65 \pm 32,66$ & $80,1 \pm 30,37$ \\
Stearic acid (C18:0) & $214,81 \pm 25,52$ & $205,03 \pm 40,13$ \\
Oleic acid (C18:1) & $629,13 \pm 122,85$ & $735,77 \pm 188,69$ \\
Linoleic acisd (C18:2) & $936 \pm 180,19$ & $789,18 \pm 165,39$ \\
Arachidic acid (C20:0) & $6,69 \pm 0,59$ & $6,65 \pm 1,30$ \\
Linolenic acid (C18:3) & $17,38 \pm 5,06$ & $16,56 \pm 6,62$ \\
Behenic acid (C22:0) & $13,23 \pm 2,55$ & $12,51 \pm 3,66$ \\
Arachidonic acid (C20:4) & $231,7 \pm 60,07$ & $246,21 \pm 45,90$ \\
Lignoceric acid (C24:0) & $15,58 \pm 3,97$ & $13,85 \pm 3,73$ \\
Eicosapentaenoic acid (C20:5) & $18,67 \pm 6,71$ & $16,88 \pm 7,37$ \\
Nervonic acid (C24:1) & $34,07 \pm 9,92$ & $36,71 \pm 7,11$ \\
Decosahexaenoic acid (C22:6) & $87,52 \pm 20,85$ & $93,43 \pm 20,08$ \\
Total & $2950,98 \pm 434,19$ & $3017,81 \pm 510,34$ \\
\hline
\end{tabular}

The data are expressed as mean \pm SEM

Fig. 4c) in the plasma concentration of sCD36 in the individuals with obesity compared to the lean controls, although the change did not reach significant level. Whereas, the plasma concentration of RBP4 of individuals with obesity was markedly elevated $(+43 \%, P=0.04$; Fig. 4b) in comparison with the lean subjects. Interestingly, there was no difference in the total plasma fatty acid concentration, as well as in particular fatty acid species between obese patients and lean controls $(P>0.05$; Table 2).

\section{Discussion}

\section{Fatty acid membrane transport}

In the previous studies, several authors have shown ${ }^{5,13-18}$ that FAT/CD36 is a major regulator of LCFA transport across the plasma membrane in various metabolically active tissues, including adipose tissue. In our study, we demonstrated an increase in the expression of FAT/CD36 in obese patients (in VAT and to a lesser extend in SAT) compared to the lean controls. Similar data, although in rats, were reported by Luiken et al. ${ }^{14}$. These authors have presented FAT/CD36 mRNA expression, which was significantly increased in epididymal adipose tissue of obese Zucker rats. According to our study and others ${ }^{14}$, we can speculate that up-regulation of FAT/CD36 gene expression may be responsible at least partially for the excessive lipid accumulation in adipose tissue. Interestingly, observed augmented expression of FAT/CD36 on different levels (mRNA and plasma membranes) in VAT compared to SAT in patients with morbid obesity may also confirm that VAT is more metabolically active tissue than SAT in this group of people. However, Bower et al. ${ }^{19}$ compared the mRNA and protein expression of FAT/CD36 in the VAT of obese Afro-American women (AAW) and Caucasian women (CAW). These authors revealed that the mRNA expression of FAT/CD36 in AAW tends to be higher compared to CAW in the VAT, which was also followed by increased uptake of LCFA in this group. Although we examined only morbidly obese Caucasian race subjects in our study, we could also observe the elevation in FAT/ CD36 plasmalemmal and mRNA expression in VAT compared with the lean group. On this bases, it is likely that the VAT of the subjects with obesity might have had a greater potential for the uptake of LCFA, similarly to other authors $^{19}$. Furthermore, we indicate increased expression of PPAR $\gamma$ together with LPL in both examined depots of adipose tissue in obese individuals. It is likely, that in our study PPAR $\gamma$, a major regulator of adipogenesis, is partially responsible for enhanced expression of FAT/CD36 and LPL, since it serves as a transcription factor for these genes in mature adipocytes ${ }^{20}$. Interestingly, we did not observe alternations in the total plasma content of fatty acids from different lipid fractions (it represents plasma fatty acids status) in morbidly obese patients (Table 2). Probably, 
it resulted from significantly increased LPL along with FAT/CD36 (especially plasmalemmal) expression in both examined compartments of adipose tissue, which compensated fatty acids dietary intake in a long-term basis by enhancing TAGs accumulation in adipose tissue. Studies conducted on mice with increased expression of LPL and high-fat diet induced obesity ${ }^{21}$ revealed protective role of this enzyme in glucose and insulin tolerance. It is in line with present study, since our patients in spite of morbid obesity were insulin sensitive and did not display symptoms of metabolic syndrome. Moreover, in the current study FABPpm mRNA expression was substantially lower in the SAT, as well as VAT in the individuals with obesity compared to the lean subjects. Similar data were noticed by Lappas et $\mathrm{al}^{22}$, in which the authors have presented that FABPpm mRNA expression in the SAT of obese (normal glucose tolerant pregnant women) has also reached significant decrease. Probably, in our case the decrease in mRNA was not followed by a decline in FABPpm expression at the protein level. Furthermore, in our study there was no change in FATP-4 mRNA and protein expression in the SAT and VAT in subjects with obesity compared to the respective tissues of the lean control. Thus, we can speculate that this protein may not be essential for fatty acid uptake in adipocytes. This notion found confirmation in a mouse model of adipocyte Fatp4 gene inactivation ${ }^{23}$, which revealed that lack of FATP-4 expression did not affect fatty acid transport into the cells. Taken together, our and others ${ }^{5}$ observations indicate that FAT/CD36 is a major LCFA protein transporter, which is responsible for augmented fatty acid uptake especially in VAT depot and subsequent accumulation of TAGs, as energy storage.

\section{Cytosolic FABPs}

FABPc stimulate not only FA desorption from biological membranes but also their cytoplasmic transport to the destination place $^{6}$. Thus, FABPc can be defined as not only the acceptors but also as a transport proteins ${ }^{24}$. In our study, there was no change in FABP4 protein expression in SAT and VAT in patients with morbid obesity compared to the lean controls. Animal studies have shown that FABP4 deficiency improved insulin resistance, lipid metabolism, atherosclerosis, and inflammatory state, under conditions of genetic or dietary obesity $^{25,26}$. Thus, it is not surprising that we did not observe any alternations in FABP4 expression in different depots of adipose tissue, since examined patients with morbid obesity were sensitive to insulin. Lack of changes in FABP4 (plasma concentration and tissue expression) and increased PPAR $\gamma$ expression in morbidly obese subjects is in agreement with studies revealing negative feedback between expression of FABP4 and PPAR $\gamma$ content in adipocytes $^{27}$. The other form of adipocyte FABPs is known as a keratinocyte lipid-binding protein or mal1
(FABP5), which is also released by macrophages and found in the skin, lung, brain, testis, and lens ${ }^{28-31}$. Based on our study, we may only hypothesize that increased expression of FABP5 in obese VAT compared with obese SAT could be due to the fact that VAT is more metabolically active than SAT in obesity. Furthermore, we cannot exclude that observed rise is gender-dependent, since the majority of examined obese subjects were women. However, additional studies are needed to explain the exact role of FABP5 in human obesity.

\section{Plasma FABPs}

In the current study, we noticed lack of changes in FABP4 plasma concentration in obese patients compared with the lean subjects. This is consistent with the results of total FABP4 expression in VAT, as well SAT tissues, since we did not noticed any alternations in morbidly obese individuals. Moreover, other authors ${ }^{32}$ have presented close correlation between plasma levels of FABP4 and its expression in adipose tissue in different fat depots, suggesting that adipose tissue is a major contributor of circulating FABP4. Furthermore, it was shown that plasma level of FABP4 can be an indicator of metabolic syndrome, type 2 diabetes mellitus, and increased lipolytic activity of adipose tissue. e.g., weight loss ${ }^{33,34}$. Our results are in agreement with the above mentioned, since patients examined in our study did not exhibit any components of metabolic syndrome in spite of morbid obesity. Therefore, our findings confirmed the hypothesis that plasma FABP4 level can serve as a biomarker of insulin resistance or metabolic syndrome.

Moreover, we revealed an upward trend for elevated plasma concentration of sCD36 in patients with obesity compared to the lean controls. Recently, it has been shown that, circulating sCD36 level was positively associated with the size of abdominal fat depots in individuals with morbid obesity ${ }^{35}$. Additionally, it was found that the level of sCD36 is associated not only with the 'unhealthy fat distribution' but also with the circulating levels of TAG. Moreover, previous studies have presented that the plasma sCD36 content is significantly higher among obese and diabetic subjects ${ }^{36,37}$. Additionally, Knöstgard et al. ${ }^{35}$ has demonstrated that adults with morbid obesity who underwent bariatric surgery presented significant weight loss and improvements in metabolic disturbances, along with a concomitant reduction in circulating sCD36. The results of our study and others ${ }^{35}$ are consistent with other findings and support an important role of sCD36 in the development of complications associated with dietinduced obesity ${ }^{38,} 39$.

Retinol binding protein (RBP4) is another peptide secreted from adipocyte and liver. In our study, we have demonstrated that RBP4 concentration was significantly elevated in the plasma of patients with obesity compared 
with non-obese group, which was consistent with previous investigation ${ }^{40}$. Similarly to our results, it was shown that increased level of RBP4 positively correlates with body mass index, as well as waist circumference ${ }^{41,42}$. There are contradictory data concerning close connection between raised values of circulating RBP4 and development of insulin resistance. However, it was indicated that increased level of RBP4 changes glucose metabolism and therefore may lead to insulin resistance ${ }^{43}$. Researchers have also demonstrated ${ }^{40,44}$ that circulating RBP4 level was linked to the concentration of plasma triacylglycerols and cholesterol in such states as obesity, type 2 diabetes, and cardiovascular implications. Thus, it means that RBP4 affects not only glucose pathway but also lipid metabolism. Probably, in our case elevated plasma levels of RBP4, as well as sCD36 (a trend) are indicators of obesity along with developing insulin resistance state and cardiovascular disorders.

For the first time we have shown the status of different fatty acid handling proteins (in plasma and adipose tissue) that are involved in adipocyte's lipid metabolism in morbidly obese patients without metabolic syndrome symptoms. Our findings indicate on FAT/CD36, as a major protein transporter enhancing FA uptake in adipocytes, especially in VAT. Moreover, in the present study we showed a relevant role of FAT/CD36, LPL, and PPAR $\gamma$ in buffering excessive fatty acid dietary intake in morbidly obese but healthy individuals. Undoubtedly, the role of FABP4, sCD36, and RBP4 as the potential biomarkers of obesity, metabolic syndrome, and cardiovascular diseases was also confirmed in our studies.

\section{Acknowledgements}

The study was funded by the Medical University of Bialystok (Grants numbers: N/ST/MN/15/001/3302 and N/ST/MN/16/001/3302).

\section{Author details}

'Department of Hygiene, Epidemiology and Ergonomics, Medical University of Bialystok, Bialystok, Poland. 'Department of Physiology, Medical University of Bialystok, Mickiewicza 2C, 15-222 Bialystok, Poland. ${ }^{3}$ Department of General and Endocrinological Surgery, Medical University of Bialystok, Bialystok, Poland

\section{Conflict of interest}

The authors declare that they have no conflict of interest.

Received: 6 April 2017 Revised: 20 October 2017 Accepted: 17 November 2017

Published online: 15 January 2018

\section{References}

1. Lebeck, J. Metabolic impact of the glycerol channels AQP7 and AQP9 in adipose tissue and liver. J. Mol. Endocrinol. 52, R165-R178 (2014).

2. Hotamisligil, G. S. Inflammation and metabolic disorders. Nature $\mathbf{4 4 4 , 8 6 0 - 8 6 7}$ (2006).

3. Cancello, R. et al. Increased infiltration of macrophages in omental adipose tissue is associated with marked hepatic lesions in morbid human obesity. Diabetes 55, 1554-1561 (2006).
4. Zhou, D. et al. CD36 level and trafficking are determinants of lipolysis in adipocytes. Faseb. J. 26, 4733-4742 (2012).

5. Coburn, C. T. et al. Defective uptake and utilization of long chain fatty acids in muscle and adipose tissues of CD36 knockout mice. J. Biol. Chem. 275, 32523-32529 (2000).

6. McArthur, M. J. et al. Cellular uptake and intracellular trafficking of long chain fatty acids. J. Lipid Res. 40, 1371-1383 (1999).

7. Fisher, R. M., Thorne, A., Hamsten, A. \& Arner, P. Fatty acid binding protein expression in different human adipose tissue depots in relation to rates of lipolysis and insulin concentration in obese individuals. Mol. Cell. Biochem. 239, 95-100 (2002).

8. Pfaffl, M. W. A new mathematical model for relative quantification in real-time RT-PCR. Nucleic Acids Res. 29, e45 (2001).

9. Gargiulo, C. E., Stuhlsatz-Krouper, S. M. \& Schaffer, J. E. Localization of adipocyte long-chain fatty acyl-CoA synthetase at the plasma membrane. J. Lipid Res. 40, 881-892 (1999).

10. Miklosz, A., Chabowski, A., Zendzian-Piotrowska, M. \& Gorski, J. Effects of hyperthyroidism on lipid content and composition in oxidative and glycolytic muscles in rats. J. Physiol. Pharmacol. 63, 403-410 (2012).

11. Glaser, C., Demmelmair, H. \& Koletzko, B. High-throughput analysis of total plasma fatty acid composition with direct in situ transesterification. PLoS One 5, e12045 (2010).

12. Folch, J., Lees, M. \& Sloane Stanley, G. H. A simple method for the isolation and purification of total lipides from animal tissues. J. Biol. Chem. 226, 497-509 (1957).

13. Bonen, A. et al. Triacylglycerol accumulation in human obesity and type 2 diabetes is associated with increased rates of skeletal muscle fatty acid transport and increased sarcolemmal FAT/CD36. Faseb. J. 18, 1144-1146 (2004).

14. Luiken, J. J. et al. Increased rates of fatty acid uptake and plasmalemmal fatty acid transporters in obese Zucker rats. J. Biol. Chem. 276, 40567-40573 (2001).

15. Luiken, J. J. et al. Changes in fatty acid transport and transporters are related to the severity of insulin deficiency. Am. J. Physiol. Endocrinol. Metab. 283, E612-E621 (2002).

16. Chocian, G. et al. High fat diet induces ceramide and sphingomyelin formation in rat's liver nuclei. Mol. Cell. Biochem. 340, 125-131 (2010).

17. Steinberg, G. R. et al. Chronic leptin administration decreases fatty acid uptake and fatty acid transporters in rat skeletal muscle. J. Biol. Chem. 277, 8854-8860 (2002).

18. Febbraio, M. et al. A null mutation in murine CD36 reveals an important role in fatty acid and lipoprotein metabolism. J. Biol. Chem. 274, 19055-19062 (1999).

19. Bower, J. F., Davis, J. M., Hao, E. \& Barakat, H. A. Differences in transport of fatty acids and expression of fatty acid transporting proteins in adipose tissue of obese black and white women. Am. J. Physiol. Endocrinol. Metab. 290, E87-E91 (2006).

20. Tontonoz, P. \& Spiegelman, B. M. Fat and beyond: the diverse biology of PPARgamma. Annu. Rev. Biochem. 77, 289-312 (2008).

21. Walton, R. G. et al. Increasing adipocyte lipoprotein lipase improves glucose metabolism in high fat diet-induced obesity. J. Biol. Chem. 290, 11547-11556 (2015).

22. Lappas, M. Effect of pre-existing maternal obesity, gestational diabetes and adipokines on the expression of genes involved in lipid metabolism in adipose tissue. Metabolism 63, 250-262 (2014).

23. Lenz, L. S. et al. Adipocyte-specific inactivation of Acyl-CoA synthetase fatty acid transport protein 4 (Fatp4) in mice causes adipose hypertrophy and alterations in metabolism of complex lipids under high fat diet. J. Biol. Chem. 286, 35578-35587 (2011).

24. Storch, J. \& Thumser, A. E. The fatty acid transport function of fatty acidbinding proteins. Biochim. Biophys. Acta 1486, 28-44 (2000).

25. Hotamisligil, G. S. et al. Uncoupling of obesity from insulin resistance through a targeted mutation in aP2, the adipocyte fatty acid binding protein. Science 274, 1377-1379 (1996).

26. Uysal, K. T., Scheja, L., Wiesbrock, S. M., Bonner-Weir, S. \& Hotamisligil, G. S. Improved glucose and lipid metabolism in genetically obese mice lacking aP2. Endocrinology 141, 3388-3396 (2000).

27. Garin-Shkolnik, T., Rudich, A., Hotamisligil, G. S. \& Rubinstein, M. FABP4 attenuates PPARY and adipogenesis and is inversely correlated with PPARY in adipose tissues. Diabetes 63, 900-911 (2014).

28. Krieg, P., Feil, S., Furstenberger, G. \& Bowden, G. T. Tumor-specific overexpression of a novel keratinocyte lipid-binding protein. Identification and 
characterization of a cloned sequence activated during multistage carcinogenesis in mouse skin. J. Biol. Chem. 268, 17362-17369 (1993).

29. De León, M. et al. Fatty acid binding protein is induced in neurons of the dorsal root ganglia after peripheral nerve injury. J. Neurosci. Res. 44, 283-292 (1996).

30. Kingma, P. B., Bok, D. \& Ong, D. E. Bovine epidermal fatty acid-binding protein determination of ligand specificity and cellular localization in retina and testis. Biochemistry 37, 3250-3257 (1998).

31. Wen, Y., Li, G. W., Chen, P., Wong, E. \& Bekhor, I. Lens epithelial cell mRNA, II. Expression of a mRNA encoding a lipid-binding protein in rat lens epithelial cells. Gene 158, 269-274 (1995).

32. $\mathrm{Xu}, \mathrm{A}$. et al. Circulating adipocyte-fatty acid binding protein levels predict the development of the metabolic syndrome: a 5-year prospective study. Circulation 115, 1537-1543 (2007).

33. Engl, J. et al. A-FABP-a biomarker associated with the metabolic syndrome and/or an indicator of weight change? Obesity 16, 1838-1842 (2008).

34. YE, W. H. et al. [Relations between serum adipocyte fatty acid-binding protein and type 2 diabetes in a community population]. Nan. Fang. Yi. Ke. Da. Xue. Xue. Bao. 31, 508-511 (2011).

35. Knøsgaard, L., Thomsen, S. B., Støckel, M., Vestergaard, H. \& Handberg, A. Circulating SCD36 is associated with unhealthy fat distribution and elevated circulating triglycerides in morbidly obese individuals. Nutr. Diabetes $\mathbf{4}$, e114 (2014).
36. Handberg, A., Levin, K., Højlund, K. \& Beck-Nielsen, H. Identification of the oxidized low-density lipoprotein scavenger receptor CD36 in plasma: a nove marker of insulin resistance. Circulation 114, 1169-1176 (2006).

37. Handberg, A. et al. Plasma sCD36 is associated with markers of atherosclerosis, insulin resistance and fatty liver in a nondiabetic healthy population. J. Intern. Med. 271, 294-304 (2012)

38. Koonen, D. P., Jensen, M. K. \& Handberg, A. Soluble CD36- a marker of the (pathophysiological) role of CD36 in the metabolic syndrome? Arch. Physiol. Biochem. 117, 57-63 (2011).

39. Love-Gregory, L. \& Abumrad, N. A. CD36 genetics and the metabolic complications of obesity. Curr. Opin. Clin. Nutr. Metab. Care 14, 527-534 (2011).

40. Lee, D. C., Lee, J. W. \& Im, J. A. Association of serum retinol binding protein 4 and insulin resistance in apparently healthy adolescents. Metabolism 56, 327-331 (2007)

41. Haider, D. G. et al. Serum retinol-binding protein 4 is reduced after weight loss in morbidly obese subjects. J. Clin. Endocrinol. Metab. 92, 1168-1171 (2007).

42. Rocha, M. et al. Association of serum retinol binding protein 4 with atherogenic dyslipidemia in morbid obese patients. PLoS One 8, e78670 (2013).

43. Muoio, D. M. \& Newgard, C. B. Metabolism: a is for adipokine. Nature 436, 337-338 (2005). England.

44. Ingelsson, E. et al. Circulating retinol-binding protein 4, cardiovascular risk factors and prevalent cardiovascular disease in elderly. Atherosclerosis $\mathbf{2 0 6}$ 239-244 (2009). 